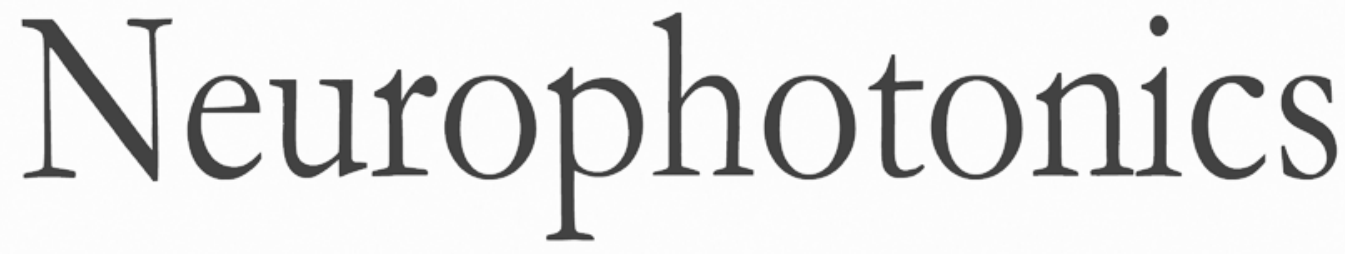

\title{
Are ventrolateral and dorsolateral prefrontal cortices involved in the computerized Corsi block-tapping test execution? An fNIRS study
}

Stefania Lancia

Vincenza Cofini

Marika Carrieri

Marco Ferrari

Valentina Quaresima 


\title{
Are ventrolateral and dorsolateral prefrontal cortices involved in the computerized Corsi block-tapping test execution? An fNIRS study
}

\author{
Stefania Lancia, Vincenza Cofini, Marika Carrieri, Marco Ferrari, and Valentina Quaresima* \\ University of L'Aquila, Department of Life, Health and Environmental Sciences, L'Aquila, Italy
}

\begin{abstract}
The Corsi block-tapping test (CBT) is an old neuropsychological test that, requiring the storage and the reproduction of spatial locations, assesses spatial working memory (WM). Despite its wide use in clinical practice, the specific contribution of prefrontal cortex (PFC) subregions during CBT execution has not been clarified yet. Considering the importance of spatial WM in daily life and the well-known role of ventrolateral$\mathrm{PFC} /$ dorsolateral-PFC (VLPFC/DLPFC) in WM processes, the present study was aimed at investigating, by a 20-channel functional near-infrared spectroscopy (fNIRS) system (including four short-separation channels), the hemodynamic response of the VLPFC/DLPFC during a computerized version of the CBT. Thirty-nine university students were asked to perform CBT standard version (CBTs), block-suppression CBT (CBTb), and control task (CBTC). A VLPFC activation during CBTs and a DLPFC activation during CBTb were hypothesized. The results of the Bayesian analysis have not shown a delineated specific activation of VLPFC/DLPFC during either CBTs or CBTb. These results together with the related ones obtained by others using fMRI are not sufficient to definitively state the role of the PFC subregions during CBT execution. The adoption of high-density diffuse optical tomography would be helpful in further exploration of the PFC involvement in spatial WM tasks. ๑ 2018 Society of Photo-Optical Instrumentation Engineers (SPIE) [DOI: 10.1117/1.NPh.5.1.011019]
\end{abstract}

Keywords: fNIRS; working memory; Corsi block-tapping test; dorsolateral prefrontal cortex; ventrolateral prefrontal cortex. Paper 17019SSRRR received Feb. 21, 2017; accepted for publication Dec. 20, 2017; published online Jan. 19, 2018.

\section{Introduction}

Everyday humans have to face a complex environment, managing, maintaining, and manipulating several different kinds of information. Even the simplest actions (e.g., remembering a telephone number until dialing it) require the competence of maintaining and using information in the mind within a short timeframe. Moreover, people and objects often change location, which leads to the need to update spatial information constantly. The cognitive function underlying those operations is defined as working memory (WM). Baddeley et al. ${ }^{1}$ divided WM into storage and executive components, introducing the most influential model of WM. The executive component, with flexible processing capacities, is responsible for information manipulation, whereas storage components (responsible for maintaining information online) are distinguished between two modality specific systems: one for phonological information and the other for visuospatial information. More specifically, the spatial system of the WM consists of two components: the visuospatial sketchpad, responsible for the passive storage of information, ${ }^{2}$ and the central executive, which controls higher-level cognitive operations, such as manipulation, update of information, dual task coordination, and inhibition. ${ }^{3,4}$ The neural substrate of the WM could be identified in a network involving the prefrontal cortex (PFC) and the posterior sensory areas (e.g., parietal cortex, occipital cortex). The role of PFC in WM processes was previously highlighted by several clinical studies showing that braindamaged patients (i.e., those with frontal lesions) have strong

*Address all correspondence to: Valentina Quaresima, E-mail: valentina quaresima@univaq.it deficits in WM tasks. ${ }^{5}$ Furthermore, supporting evidence comes from imaging studies that investigated the overlap between the activation of the prefrontal and the parietal regions during spatial WM tests performed by healthy participants and the location of the lesion in patients with visuospatial disorders. ${ }^{6,7}$ Some of the earliest neuroimaging studies on WM raised questions regarding the material-type effects in the prefrontal areas. Although some studies emphasized the verbal WM left lateralization and spatial WM right lateralization, many studies have not found lateralization within PFC during verbal and/or spatial WM tasks. ${ }^{8}$ Those studies supported the right/left lateralization in relation to the executive demand of the task rather than in relation to the material-type. Furthermore, even though neuroimaging studies have provided evidence that the PFC is crucially involved in WM, the differential contributions of its subregions are still a matter of debate. On one hand, there is a perspective that emphasizes the ventrolateral-PFC (VLPFC) involvement in simple short-term memory operations (e.g., the maintenance or rehearsal of WM contents) and dorsolateral-PFC (DLPFC) involvement in higher-level executive processes (strategic processing, monitoring, manipulation of WM content, inhibition of irrelevant information).$^{9-13}$ On the other hand, a different perspective shows VLPFC involvement in WM processing of information on objects and DLPFC involvement in processing spatial WM information, suggesting that the PFC subregions underlie the processing of several WM content. ${ }^{14-16}$

Taking into consideration the importance of spatial WM in daily life, several neuropsychological tests were adopted to 


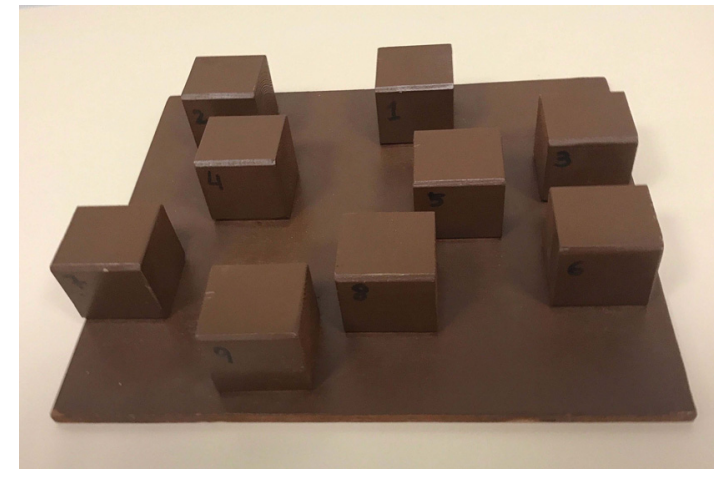

Fig. 1 The standard version of the Corsi block-tapping board. The base of wood board was $29 \times 24 \mathrm{~cm}$ and the 9 cubes measured $4 \mathrm{~cm}$.

assess individual temporary storage capability, such as the Corsi block-tapping test (CBT). ${ }^{17}$ Although this test was introduced almost 50 years ago, the CBT is still used in clinical settings in its traditional version consisting of nine cubes irregularly positioned on a board (Fig. 1).

In its standard version, the examiner taps the cubes on the board in sequences of increasing length and, immediately after each tapped sequence, the subject is asked to reproduce it as the examiner has done. Starting with sequences of three cubes, the number of blocks within the sequences gradually increases by one and progresses until the subject makes two consecutive errors. The original CBT was developed as a nonverbal task to assess incidental learning in patients with epilepsy following temporal lobe excision. ${ }^{18}$ Based on those investigations, it was inferred that the left medial temporal lobe mediated memory consolidation of verbal sequences, whereas the right medial temporal region mediated memory consolidation of spatial (block-tapping) sequences. ${ }^{18}$ The Corsi findings were so persuasive that the CBT was considered as one of the pre-eminent measures of the spatial WM. ${ }^{19}$

Despite the relevance of this test in clinical practice, to the best of our knowledge, only few neuroimaging studies have investigated healthy subjects during the CBT execution. In a positron emission tomography (PET) study, Bor et al. ${ }^{9}$ showed an increase of glucose metabolism in the VLPFC. More recently, the functional magnetic resonance imaging (fMRI) study by Toepper et al. ${ }^{13}$ has demonstrated that the execution of a computerized version of the CBT activated the frontal areas, suggesting the role of these areas in executive processes that control the update of positional information during WM processing. ${ }^{12}$ In addition, Toepper et al. ${ }^{13}$ used the block suppression test paradigm developed by Beblo et al. ${ }^{20}$ This paradigm, based on the original Corsi block-tapping test version, requires a higher-level of executive control to actively inhibit the distractors appearing during the presentation of the target cubes (the cubes to be memorized). In fact, during the suppression of the distractors (inhibition of irrelevant information), Toepper et al. ${ }^{13}$ observed an activation of the DLPFC.

In the last 20 years, another neuroimaging technique, the functional near-infrared spectroscopy (fNIRS), has been largely applied in cognitive neuroscience. ${ }^{21,22}$ fNIRS is a noninvasive vascular-based technique that measures concentration changes in oxygenated $\left(\mathrm{O}_{2} \mathrm{Hb}\right)$ and deoxygenated $(\mathrm{HHb})$ hemoglobin of the cerebral microcirculation blood vessels. The coupling between neuronal activity and cerebral blood flow is fundamental to brain function, and fNIRS relies on this coupling to infer changes in neural activity, which is reflected by the blood oxygenation changes of the activated cortical region (i.e., the increase in $\mathrm{O}_{2} \mathrm{Hb}$ and concomitant decrease in $\mathrm{HHb}$ ). ${ }^{23,24}$

For this reason, a combined use of the fNIRS technique and the CBT could be a valid tool to better clarify what is the specific contribution of the PFC subregions (i.e., VLPFC and DLPFC) in the WM. Therefore, considering the controversial role of these PFC subregions in the WM and the features of the fNIRS technique, this study aims to investigate, using a 20-channel fNIRS system [including four short-separation (SS) channels], the cortical hemodynamic changes in the VLPFC/DLPFC during a computerized version of the CBT. This new computerized version of the CBT, consisting of the standard CBT (CBTs), block-suppression CBT (CBTb), and a control task (CBTc), was implemented in this study. Considering that (1) CBT execution requires the maintenance of positional information and (2) $\mathrm{CBTb}$ execution requires the ability to inhibit a response (a higher-level cognitive operation), it was hypothesized that VLPFC would be bilaterally activated during CBTs, whereas DLPFC would be bilaterally activated during CBTb.

\section{Materials and Methods}

\subsection{Participants}

Thirty-nine right-handed university students aged between $23.9 \pm 3.1$ years-level of education: $14.8 \pm 1.7$ years-participated in the study. Only subjects who, in a preliminary familiarization session, correctly remembered a five-cube sequence (spatial span $\geq 5$ ) were included in the study (see Sec. 2.4). This group consisted of 19 women (age $=23.1 \pm 3.1$ years) and 20 men (age $=24.6 \pm 2.8$ years). The inclusion criterion assumed no history of neurological or psychiatric diseases (including substance abuse or dependence). This was determined by the participants' responses to a questionnaire in which they were asked to indicate any previous or current diseases. Testing always took place at the same time of the day. All participants declared that they had adequate sleep ( 6 to $8 \mathrm{~h}$ ) and had not recently (over the last 2 weeks) travelled across time zones and had not drunk coffee or smoked cigarettes before testing. To exclude left-handed subjects, all participants completed the Edinburgh Handedness Inventory ${ }^{25}$ assessing hand dominance. According to the tenets of the latest Declaration of Helsinki, written and informed consent was obtained from each participant prior to the recording after a full explanation of the protocol and the noninvasiveness of the study. The study was approved by the University Ethics Committee.

\subsection{Corsi Block-Tapping Test-Computerized Version}

Stimulus material consisted of nine gray cubes positioned as in the original block formation of the Corsi board (Fig. 1). The cubes of the computerized CBT version had the same size as the cubes of the wood board. The background color was black and the target cubes (the cubes to be memorized) were highlighted in red. This implemented CBT computerized version included three blocks randomly presented to all participants: the CBTs, the CBTb, and the CBTc (Fig. 2). In both CBTs and CBTb, each sequence included a storage, a maintenance, and a reproduction phase. During the storage phase, target stimuli were presented on the board. During the maintenance phase, the subjects were requested to maintain the target stimuli presented in the storage phase. During the reproduction phase, subjects were asked to 
reproduce the sequence making from three to six sequential decisions (according to the sequence length). Four different sequences (three, four, five, and six cubes) were implemented, and for each length, five sequences were randomly presented (five sequences of three cubes, five sequences of four cubes, five sequences of five cubes, and five sequences of six cubes). Only the forward reproduction was required. On the computer screen, the sequences were preceded by a white fixation cross lasting $500 \mathrm{~ms}$; each target cube was presented on the Corsi board once in a sequence for $1 \mathrm{~s}$. The interval between the target cubes was $1 \mathrm{~s}$, during which no target cubes (gray cubes) were presented on the board. Only in the case of CBTb, target stimuli were presented together with distractor cubes (gray cubes with red outline). Sequences were followed by a $6 \mathrm{~s}$ maintenance period in which the computer screen was black. After the maintenance phase, participants had to decide between two alternative response options for each presented target cube of the sequence, starting with the first one. Two red cubes were displayed on the Corsi board, labeled with an "S" letter ("sinistra" in Italian, "left" in English) for the cube, which was located more left in relation to a second one, which was labeled with a "D" letter ("destra" in Italian, "right" in English). One of these cubes represented the first target location of the sequence, whereas the other one was a randomly chosen alternative (Fig. 2). Subjects were requested to answer by pressing the "S" or "D" key on a keypad $(10 \times 8 \mathrm{~cm})$ placed under their right hand. Directly after this first decision, regardless whether correct or incorrect, two new red cubes were displayed representing two alternative response options for the location of the second cube of the sequence. This procedure remained the same for every target cube of each sequence in the CBTs and the CBTb blocks. Subjects' correct and incorrect responses for each sequence and task were recorded for the behavioral analysis. During CBTc block, a simple motor task, requiring one to press the key corresponding to the highlighted letter ("S" or "D"), was presented. A commercially available software package (SuperLab Pro Edition 4.5 Executable, Cedrus Corporation, Canada) was used for protocol implementation.

\section{3 fNIRS Instrumentation and Data Processing}

A 20-channel continuous wave fNIRS system (OxyMon Mk III, Artinis Medical Systems, The Netherlands) was employed to map the changes in $\mathrm{O}_{2} \mathrm{Hb}$ and $\mathrm{HHb}$ over the bilateral PFC. This device measures changes in light attenuation at two wavelengths, 764 and $856 \mathrm{~nm}$. The $\mathrm{O}_{2} \mathrm{Hb} / \mathrm{HHb}$ concentration changes (expressed in $\Delta \mu \mathrm{M}$ ), obtained by using the modified BeerLambert law, ${ }^{26,27}$ are displayed in real time. Eight optical fiber bundles (length: $3.15 \mathrm{~m}$; diameter: $4.5 \mathrm{~mm}$ ) were utilized to carry out the light to the left and the right PFC (four for each hemisphere), whereas 10 optical fiber bundles of the same size (five for each hemisphere) were utilized to collect the light emerging from the same cortical areas. The illuminating and collecting bundles were assembled into a specifically designed flexible probe holder ensuring that the position of the 18 optodes, relative to each other, was firmly fixed. The probe holder consisted of two mirror-like units $(9.7 \times 8.9 \mathrm{~cm}$ each) held together, along the longest side, by three flexible junctions. In 16 of the 20 measurement points, the detector-illuminator distance was set at $3.5 \mathrm{~cm}$, whereas in four measurement points, the detector-illuminator distance was set at $1 \mathrm{~cm}$ (short-separation channels or SS channels). The optodes were inserted into a polyoxymethylene probe holder by connectors. The probe holder was appropriately placed over the head in order to include the underlying PFC. In particular, the two frontopolar fiber bundles collecting light at the bottom of the holder were centered (according to the International 10-20 system for the electroencephalography electrode placement) on the Fp1 and Fp2 locations for the left and right side, respectively. The Montreal
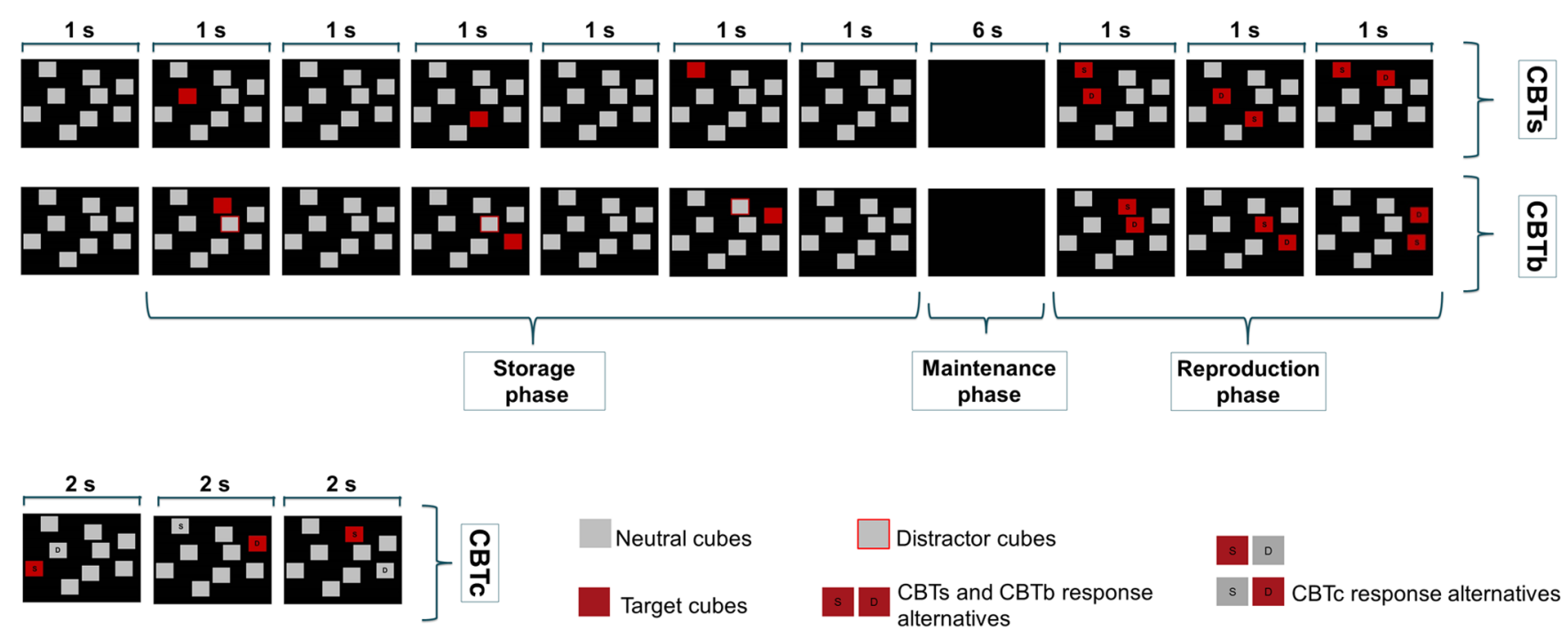

Fig. 2 Representation of a three-cube sequence during the CBTs, the CBTb, and the CBTc. During CBTs and CBTb, each target cube was usually presented on the computer screen for $1 \mathrm{~s}$. The interval between target cube presentations was $1 \mathrm{~s}$, during which a no-target cube was shown on the screen. The storage phase was followed by a black screen lasting $6 \mathrm{~s}$. The presentation time on the screen during CBTc was $2 \mathrm{~s}$. Storage and reproduction phases of the CBTs and the CBTb are illustrated. In the reproduction phases of CBTs and CBTb and during the CBTc, some cubes were labeled with "S" ("sinistra" in Italian, "left" in English) for the cube that was located more left in relation to the second one, which was labeled with "D" ("destra" in Italian, "right" in English) located more right (see Corsi block-tapping testcomputerized version section for more details). 


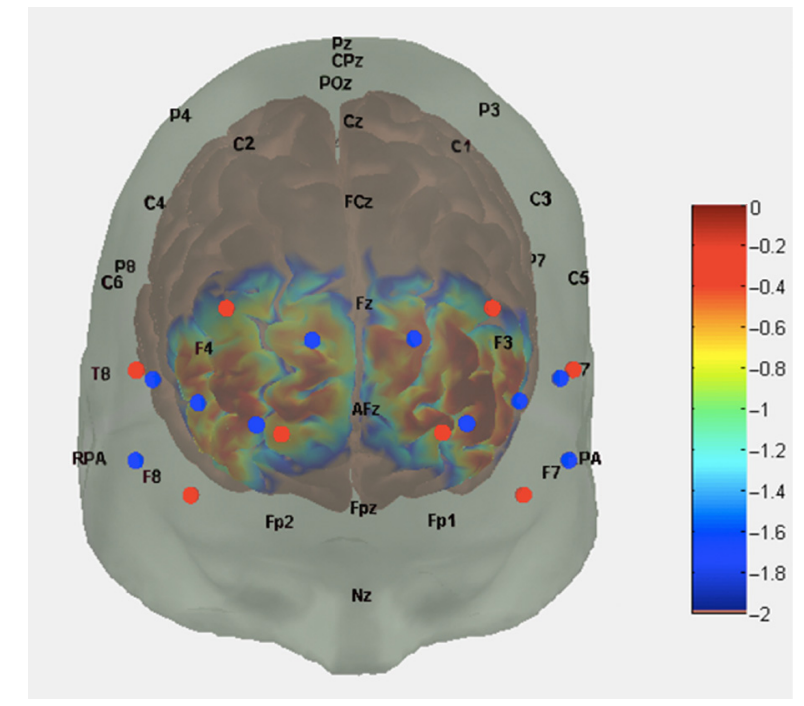

Fig. 3 Map of sensitivity profile of the 16 measurement points and 4 short-separation channels. The probe layout was registered and anchored on the digital brain adult atlas (Colin27), and sensitivity profile of the 16 measurement points and 4 short-separation channels is represented. Red and blue circles represent the illuminators and detectors, respectively. The color scale depicts the sensitivity profile from -2 (low sensitivity) to 0 (high sensitivity). See Sec. 2 for details.

Neurological Institute coordinates of the optodes and the relative 16 measurement points were calculated using a probe placement method $^{28}$ based on a physical model of the ICBM152 head surface. ${ }^{29}$ For the details of the procedure, see Ref. 30 The resulting matching Brodmann areas (BAs) of the 16 measurement points were BA 9 (measurement points: 6, 5, 13, 14), BA 45 (measurement points: $4,7,8,12,15,16$ ), and BA 46 (measurement points: 1, 2, 3, 9, 10, 11). Moreover, these BAs correspond to three major subregions of the PFC: the DLPFC (BAs 9 and 46) and the VLPFC (BA 45). A template of the probe layout was imported in AtlasViewerGUI, a part of fNIRS analysis package Homer2 (Massachusetts General Hospital/Harvard Medical School, Massachusetts), ${ }^{31,32}$ that contains "Colin27," a digital brain adults' atlas commonly used in MRI studies. A sensitivity profile map of the 16 measurement points and the four SS channels layout was created by Atlas ViewerGUI (Fig. 3).

The probe holder was fixed over the head by a velcro brand fastener, adaptable to the individual size and shape of the head. This flexible probe holder and its position on the head provided a stable optical contact with the scalp for all the optodes. The accuracy of the contact between the optodes and the scalp was verified at the end of the protocol. The pressure created by the velcro brand fastener was adequate to induce a partial transient blockage of the skin circulation during the fNIRS study, as witnessed by the presence of 18 well-defined circles over the PFC skin (i.e., depressed cutaneous areas corresponding with the location of the 18 optodes). The 18 circles over the forehead skin started to disappear 15 to $20 \mathrm{~min}$ after the end of the protocol. The adopted procedure suggests that a consistent reduction of forehead skin blood flow was occurring under the 18 optodes as a result of this approach. ${ }^{33}$ The $\mathrm{O}_{2} \mathrm{Hb} / \mathrm{HHb}$ data from the 20 measurement points, which are defined as the midpoint of the corresponding detector-illuminator pairs, were acquired at $10 \mathrm{~Hz}$. During the data collection procedure, the signal quality as well as the absence of movement artifacts was verified. The subject's heart rate (HR) was monitored by a pulse oximeter (N-600, Nellcor, Puritan Bennett, St. Louis, Missouri) with the sensor clipped to the index finger of the left hand.

The data processing was carried out using some of the Homer2 NIRS processing package functions ${ }^{31}$ based on MATLAB (Mathworks, Natick, Massachusetts). For every subject, channels with a very low optical intensity were discarded from the analysis using the function enPruneChannels. The remaining raw optical intensity data were then converted into changes in optical density (OD). Motion artifacts were corrected applying the "wavelet motion correction" method implemented into Homer2 (iqr parameter set to 0.1), which is based on the method developed by Molavi and Dumont. ${ }^{34}$ Then, the corrected OD data were converted into concentration changes using the modified Beer-Lambert's law. The hemodynamic response for each task was recovered using a general linear model (GLM) approach (with the hmrDeconvHRF_DriftSS function), which simultaneously regressed the SS channel signals to correct for physiological noise contamination. A set of Gaussian functions with standard deviation (SD) of $3 \mathrm{~s}$ and with their means separated by $2 \mathrm{~s}$ was used as temporal basis functions in an interval between 2 and $18 \mathrm{~s}$ before and after the starting of three-cube sequences, 2 and $22 \mathrm{~s}$ before and after the starting of four-cube sequences, 2 and $26 \mathrm{~s}$ before and after the starting of five-cube sequences, and 2 and $30 \mathrm{~s}$ before and after the starting of six-cube sequences. ${ }^{35}$ For each standard channel, the SS channel signal with the greatest correlation was chosen and its signal was added in the design matrix of the GLM. The iterative weighted least square method proposed by Barker et al. ${ }^{36}$ was selected for solving the GLM matrix equation. This produced 12 hemodynamic response functions (HRFs), one per sequence and task type (i.e., three-cube sequence CBTs, three-cube sequence CBTb, three-cube sequence $\mathrm{CBTc}$, four-cube sequence CBTs, four-cube sequence $\mathrm{CBTb}$, four-cube sequence CBTc, five-cube sequence CBTs, fivecube sequence $\mathrm{CBTb}$, five-cube sequence $\mathrm{CBTc}$, six-cube sequence $\mathrm{CBTs}$, six-cube sequence $\mathrm{CBTb}$, and six-cube sequence $\mathrm{CBTc}$ ), for each channel and for each subject.

\subsection{Experimental Design}

Prior to the study, participants were informed about the procedures and familiarized with the protocol. The familiarization phase was carried out 3 days before the study. During this phase, each subject's spatial span was assessed through the original wood board of the CBT (Fig. 1) and compared with the spatial span resulting from the computerized CBT version. Spatial span averages about 5 for normal human subjects, ${ }^{37}$ so in order to make the sample uniform, only subjects with spatial span $\geq 5$ were recruited for this study. The accordance between the original and the computerized versions allowed to proceed with the present protocol. According to this criterion, eight subjects were excluded.

The study was carried out in a quiet and dimly lit room. Participants were asked to sit on a comfortable high-backed chair in front of a 17" PC monitor placed at a distance of $70 \mathrm{~cm}$, and to place their right hand on the keypad with the forefinger and the middle finger upon the " $\mathrm{S}$ " and the " $\mathrm{D}$ " keys, respectively.

The CBT protocol included three blocks randomly presented across participants. Prior to the presentation of each block, visual instructions were given to subjects. Each block consisted of a 


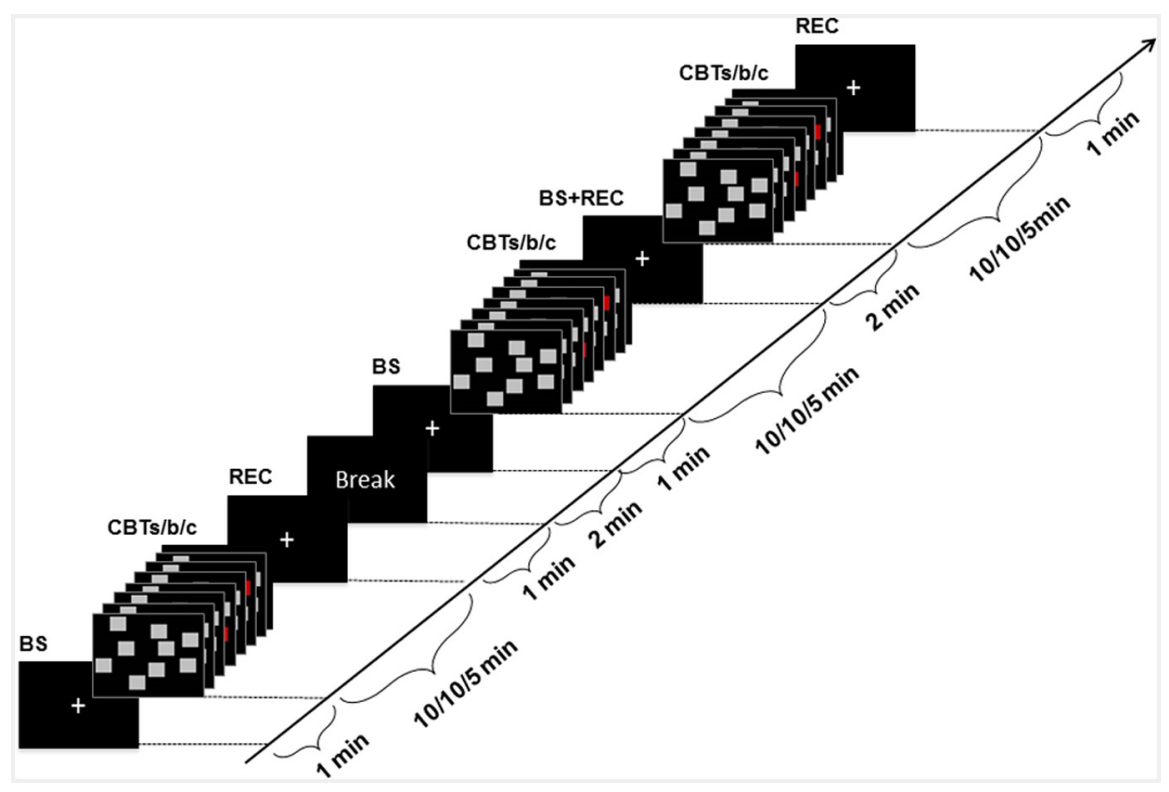

Fig. 4 Schematic representation of the protocol. BS, baseline period; CBTs/b/c, Corsi block-tapping test standard/block-suppression/control task; REC, recovery period.

baseline period $(1 \mathrm{~min})$, a task period $(10 \mathrm{~min}-\mathrm{CBTs} / 10 \mathrm{~min}-$ $\mathrm{CBTb} / 5 \mathrm{~min}-\mathrm{CBTc})$, and a recovery period $(1 \mathrm{~min})$. Specifically, during the baseline period, the participants were asked to relax while observing a white fixation cross presented on a black screen in order to get fNIRS signals as stable as possible. Between the first and the second presented blocks, a 2-min period of break was given (see Fig. 4). Subjects were asked to remain seated limiting their movements and, in the meanwhile, they were allowed to talk to the examiners.

In order to evaluate the perceived physical and/or psychological discomfort caused by the probe holder during the protocol, subjects completed a visual analogue scale (VAS), ${ }^{38}$ rating the average perceived discomfort by making a mark somewhere on a $100 \mathrm{~mm}$ line, which indicates the discomfort intensity (where 0 to $4 \mathrm{~mm}$ can be considered no discomfort; 5 to $44 \mathrm{~mm}$, mild discomfort; 45 to $74 \mathrm{~mm}$, moderate discomfort; and 75 to $100 \mathrm{~mm}$, severe discomfort). In order to evaluate the "state anxiety," subjects completed the 20-items of the STAIForm Y-1 before and after the protocol. ${ }^{39}$

\subsection{Data Analysis and Statistics}

For the behavioral data, the subjects' number of errors (i.e., performance) was calculated in CBTs, CBTb, and CBTc for each sequence. The behavioral data were expressed as means \pm SDs.

The mean values of the HR changes (analyzed as percentage of control) were calculated from the beginning of each sequence (at $0 \mathrm{~s}$ ) until the end of each sequence (18 $\mathrm{s}$ for the three-cube sequences; $22 \mathrm{~s}$ for the four-cube sequences; $26 \mathrm{~s}$ for the fivecube sequences; and $30 \mathrm{~s}$ for the six-cube sequences) for each task and subject. Both the maximum/minimum values of the $\mathrm{O}_{2} \mathrm{Hb} / \mathrm{HHb}$ changes and the $\mathrm{HR}$ mean values of the tasks and sequences periods were normalized to their baseline periods, calculated over the last $2 \mathrm{~s}$ before the starting of each sequence (i.e., from -2 to $0 \mathrm{~s}$ ). This procedure permitted one to remove the basal signal from the maximum/minimum values of the $\mathrm{O}_{2} \mathrm{Hb} / \mathrm{HHb}$ hemodynamic responses (i.e., obtaining normalized task periods) and to bring all the traces at a zero-starting value. The HR values underwent the same "correction for the baseline" procedure, for coherence with the hemodynamic data processing. The HR values were statistically analyzed in order to evaluate potential HR changes during each block execution and/or between the four sequences. However, it is important to mention that the maximum/minimum values of the $\mathrm{O}_{2} \mathrm{Hb} / \mathrm{HHb}$ changes were calculated from the HRFs corrected for the physiological noise contaminations.

The maximum/minimum values of the $\mathrm{O}_{2} \mathrm{Hb} / \mathrm{HHb}$ changes of the HRFs were calculated from the beginning of each sequence (at $0 \mathrm{~s}$ ) until the end of each sequence (18 $\mathrm{s}$ for the three-cube sequences; $22 \mathrm{~s}$ for the four-cube sequences; $26 \mathrm{~s}$ for the five-cube sequences; and $30 \mathrm{~s}$ for the six-cube sequences), for each task, measurement point, and subject. Data were analyzed according to the Bayesian approach using the free-statistical software JASP 08.3.1. (JASP Team, 2017). The results of these analyses were interpreted using the guidelines set out by Wagenmakers et al., ${ }^{40}$ who consider a $\mathrm{BF}_{10}$ value in the range 3 to 10 as moderate evidence for the alternative hypothesis $(\mathrm{H} 1)$, a $\mathrm{BF}_{10}$ value in the range 10 to 30 as strong evidence for $\mathrm{H} 1$, a $\mathrm{BF}_{10}$ value in the range 30 to 100 as very strong evidence for $\mathrm{H} 1$, and $\mathrm{BF}_{10}$ value greater than 100 as extreme evidence for H1. The Student's Bayesian paired samples $t$ test was conducted to investigate differences in the anxiety state (STAI-Form Y-1) before and after the protocol. Bayes factor of null versus alternative $\left(\mathrm{BF}_{01}\right)$ was reported. The Bayesian repeated measures analysis of variance (Bayes RM-ANOVA) was used for the performance, the HR mean values, and the maximum/minimum values of the $\mathrm{O}_{2} \mathrm{Hb} / \mathrm{HHb}$ changes, in order to investigate the effect of the tasks and/or sequences on the overall PFC activation. Specifically, the Bayes RM-ANOVA for the performance included two factors: sequence (four levels) and task (two levels); the Bayes RM-ANOVA for the HR included two factors: sequence (four levels) and task (three levels). In addition, posthoc analyses were conducted in order to investigate differences in subjects within tasks and sequences. The Pearson's correlation coefficient was calculated for evaluating the relation between the $\mathrm{HR}$ and the maximum/minimum $\mathrm{O}_{2} \mathrm{Hb} / \mathrm{HHb}$ changes during the CBTs, CBTb, and CBTc execution. The Bayes RM-ANOVA for 
the maximum/minimum values of the $\mathrm{O}_{2} \mathrm{Hb} / \mathrm{HHb}$ included three factors: measurement points (eight levels), hemisphere (two levels), sequence (four levels), and task (three levels). Separate Bayes RM-ANOVAs for each task were conducted, including within the model, the factors showing extreme evidence in the previous analyses (measurement points, sequences), in order to investigate potential differences between factors within tasks. Furthermore, in order to investigate trends and patterns of the hemodynamic responses across specific sequences eliciting PFC activation and the activations during specific sequences in the measurement points, posthoc statistical analyses were conducted. For all Bayes RM-ANOVAs, the "inclusion Bayes factors across matched models" for each component of interest in turn were reported as $\mathrm{BF}_{\mathrm{I}}$. For the posthoc analysis and the correlation analysis, the Bayes factors were reported as $\mathrm{BF}_{10}=1 / \mathrm{BF}_{01}$.

\section{Results}

The behavioral data analysis revealed the following main results. The mean subjective rating of the perceived physical and/or psychological discomfort caused by the probe holder (VAS) during the CBT protocol was $47.3 \pm 16.5$. There were no differences in the anxiety state before $(32.9 \pm 4.2)$ and after $(33.4 \pm 4.3)$ the protocol; the Bayes factor $\left(\mathrm{BF}_{01}=4.756\right)$ was in favor of the null hypothesis. For the CBTs, the performances were: $0.15 \pm 0.36$ for the three-cube sequence, $0.38 \pm$ 0.63 for the four-cube sequence, $1.25 \pm 1.14$ for the five-cube sequence, and $1.82 \pm 1.02$ for the six-cube sequence. For the $\mathrm{CBTb}$, the performances were: $0.28 \pm 0.85$ for the three-cube sequence, $0.51 \pm 0.96$ for the four-cube sequence, $1.15 \pm 1.11$ for the five-cube sequence, and $1.71 \pm 1.52$ for the six-cube sequence. For the CBTc, no errors were done. The performance analysis has provided that the inclusion of the main effect of the sequences was strongly favored $\left(\mathrm{BF}_{\mathrm{I}}=8.981 \times 10^{24}\right)$, whereas the inclusion of the task effect $\left(\mathrm{BF}_{\mathrm{I}}=0.126\right)$ and the tasksequence interaction was not supported $\left(\mathrm{BF}_{\mathrm{I}}=0.060\right.$; Fig. 5). The Bayes RM-ANOVA analysis for the HR has revealed strong evidence to include only the main effect of the task $\left(\mathrm{BF}_{\mathrm{I}}=\right.$ 1824.114). The posthoc comparisons between CBTs and CBTb reported extreme evidence for the null hypothesis $\left(\mathrm{BF}_{10}=\right.$ $0.121)$ while Bayesian factors showed evidence for the alternative hypothesis between CBTs and CBTc $\left(\mathrm{BF}_{10}=304.976\right)$ and between $\mathrm{CBTb}$ and $\mathrm{CBTc}\left(\mathrm{BF}_{10}=85.996\right)$. Bayesian analysis did not provide evidence for correlation between the $\mathrm{HR}$ and the maximum/minimum $\mathrm{O}_{2} \mathrm{Hb} / \mathrm{HHb}$ changes data. The only

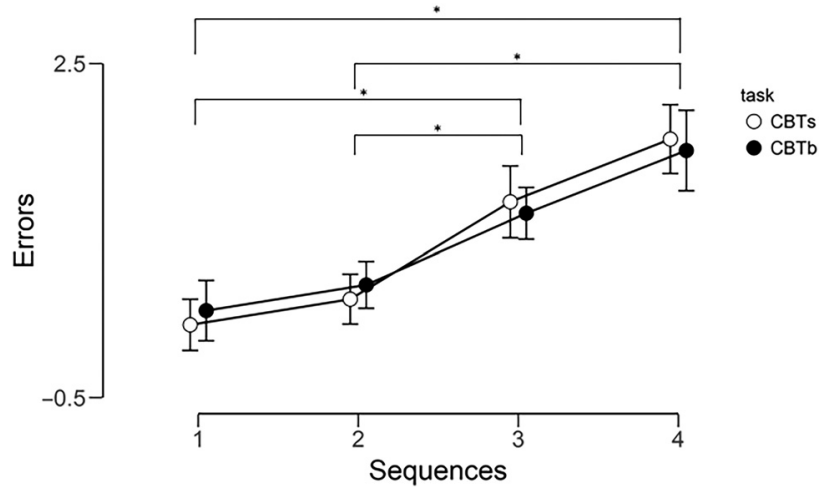

Fig. 5 Results of the posthoc comparison for the performance among the four sequences. Results are shown as mean errors per sequence length with $95 \%$ credible intervals. ${ }^{*}, \mathrm{BF}_{10}>100$. evidence was found for the $\mathrm{HR}$ and minimum $\mathrm{HHb}$ changes within the six-cube sequence during CBTb $(r=0.52$; $\left.\mathrm{BF}_{10}=50.904\right)$.

The grand average of the hemodynamic responses over the 16 measurement points, during the execution of the four sequences (sequences of 3, 4, 5, and 6 cubes), is reported (Fig. 6). The Bayes RM-ANOVAs of the fNIRS data have revealed the following main results.

The results of the analysis, made considering the maximum values of the $\mathrm{O}_{2} \mathrm{Hb}$ changes, have demonstrated that the strongest model included main effects of the measurement point $\left(\mathrm{BF}_{\mathrm{I}}=3.485 \times 10^{43}\right)$, the sequence $\left(\mathrm{BF}_{\mathrm{I}}=6.446 \times 10^{9}\right)$, and the task $\left(\mathrm{BF}_{\mathrm{I}}=2.441 \times 10^{18}\right)$. The results of the analysis, made considering the minimum values of the $\mathrm{HHb}$ changes, have demonstrated evidence for an effect of the measurement point $\left(\mathrm{BF}_{\mathrm{I}}=1.429 \times 10^{16}\right)$, the sequence $\left(\mathrm{BF}_{\mathrm{I}}=4.539 \times 10^{11}\right)$, and the task $\left(\mathrm{BF}_{\mathrm{I}}=4.743 \times 10^{24}\right)$.

The further Bayes RM-ANOVAs analyses, conducted to investigate the hemodynamic responses across specific sequences and measurement points, have revealed evidence to include the main effects of the measurement point $\left(\right.$ all $\left.\mathrm{BF}_{\mathrm{I}}>100\right)$ and the sequence (all $\mathrm{BF}_{\mathrm{I}}>100$ ) for CBTs and $\mathrm{CBTb}$ and the measurement point for CBTc (all $\mathrm{BF}_{\mathrm{I}}>100$ ).

The posthoc comparisons for sequences have provided evidence for the alternative hypothesis between all sequences within CBTs and CBTb (all $\mathrm{BF}_{10}>100$ ). The posthoc comparisons for measurement points have not provided evidence for the alternative hypothesis between all sequences within CBTs and $\mathrm{CBTb}$. For example, in the case of the most demanding sequence (six-cube), most of the $\mathrm{BF}_{10}$ were found less than 1 . The strongest evidence was found for the comparison between measurement points 1 and 14 for $\mathrm{HHb}$ values within $\mathrm{CBTb}$ $\left(\mathrm{BF}_{10}=69.978\right)$, while no evidence was found for $\mathrm{O}_{2} \mathrm{Hb}$ $\left(\mathrm{BF}_{10}=0.639\right)$.

\section{Discussion}

The CBT, introduced almost 50 years ago, is widely used in clinical settings to assess spatial WM. To the best of our knowledge, this is the first time in which a computerized version of the CBT with the noninvasive fNIRS investigation of the PFC hemodynamic changes has been utilized with the aim of clarifying what is the specific contribution of the VLPFC and DLPFC in the spatial WM.

The behavioral data analysis has shown a significant difference in the number of errors depending on the sequence length both on CBTs and CBTb, highlighting the consistent cognitive demand of the two tasks at the five-cube and six-cube sequences. Although the Bayes RM-ANOVA analysis for the maximum/minimum values of $\mathrm{O}_{2} \mathrm{Hb} / \mathrm{HHb}$ changes has revealed task, sequence, and measurement point as main effects, the subsequent posthoc analyses have not provided clear evidence for supporting our hypothesis. In fact, even if the sequence has shown a meaningful impact on subject hemodynamic changes (during the five-cube and six-cube sequences), during task execution, no involvement of specific measurement points (i.e., specific PFC regions) was found. Therefore, these results suggest that both VLPFC and DLPFC are not specifically activated (as simultaneous $\mathrm{O}_{2} \mathrm{Hb}$ increase and $\mathrm{HHb}$ decrease), during the CBTs and the CBTb.

Spatial WM has been largely investigated by fNIRS using different tests in healthy subjects and patients. ${ }^{41-44}$ Interestingly, a recent study by McKendrick et al. ${ }^{43}$ found a nonlinear increase 
(a)
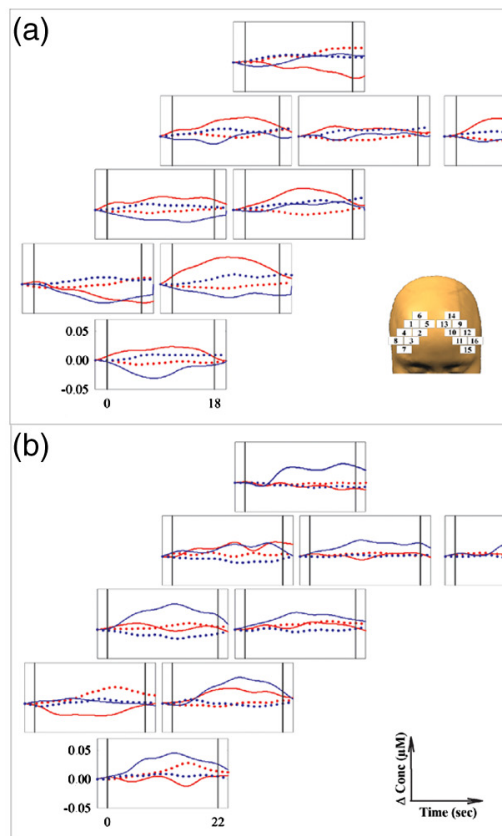
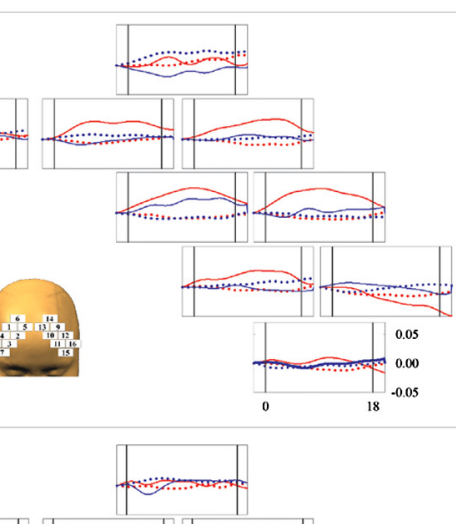

Fig. 6 Grand average $(n=39)$ of the $\mathrm{PFC} \mathrm{O}_{2} \mathrm{Hb}$ (solid lines) and $\mathrm{HHb}$ (dotted lines) changes in response to the standard Corsi block-tapping test (red) and the Corsi block-tapping test block-suppression (blue) during the four sequence length. (a) Three-cube sequence; (b) four-cube sequence; (c) five-cube sequence; and (d) six-cube sequence. The layout of the 16 fNIRS measurement points over the right (numbers from 1 to 8 ) and left (numbers from 9 to 16) hemisphere is graphically represented. The vertical solid lines limit the duration of the task execution of each sequence.

in left DLPFC and a right VLPFC after an increased exposure to WM training. In general, in most of the fNIRS spatial and nonspatial WM studies, the amplitude of the $\mathrm{O}_{2} \mathrm{Hb} / \mathrm{HHb}$ changes was not reported. Among those ones in which the amplitude of $\mathrm{O}_{2} \mathrm{Hb} / \mathrm{HHb}$ changes was reported, only in few, the standard concentration units were utilized; the range varied from 0.1 to $0.3 \mu \mathrm{M} .^{44-46}$ Therefore, the changes in response to the CBT of the present study were expected to be in the order of magnitude of 0.1 to $0.4 \mu \mathrm{M}$. The fact, that in the present study, no specific activation was found could not be attributable to the limited sensitivity of the utilized fNIRS instrumentation. The OxyMon Mk III, equipped with laser light sources and high sensitivity avalanche photo diodes, provides $\sim 0.001 \mathrm{SD}$ in OD at a total of $\sim 6 \mathrm{OD}$ at $10-\mathrm{Hz}$ measurement frequency. The instrumentation is capable to detect reliably cortical $\mathrm{O}_{2} \mathrm{Hb} / \mathrm{HHb}$ changes up to $0.05 \mu \mathrm{M}$. This concentration change corresponds to about $0.1 \%$ of the cortical total hemoglobin concentration, estimated to be around $60 \mu \mathrm{M}^{47}$

Considering that only in one fMRI study, Toepper et al. ${ }^{13}$ carried out during the execution of a computerized version of the CBTs/CBTb, activation of the VLPFC/DLPFC was observed, the previous fMRI and the present findings need to be discussed more in depth. These inconsistent results could be related to the different functional neuroimaging techniques adopted in the two separate studies. It is well known that fNIRS signals have a significantly weaker signal-to-noise ratio but they are nonetheless often highly correlated with fMRI measurements. ${ }^{48,49}$ However, sometimes, the fNIRS spatial resolution does not suffice in answering the questions of interest. Recently, Buxton, ${ }^{50}$ reviewing the physics of fMRI, suggested that "the complexity of the blood-oxygen-level dependent (BOLD) response means that it is difficult to attach a quantitative interpretation to BOLD measurements alone." In fact, the magnitude
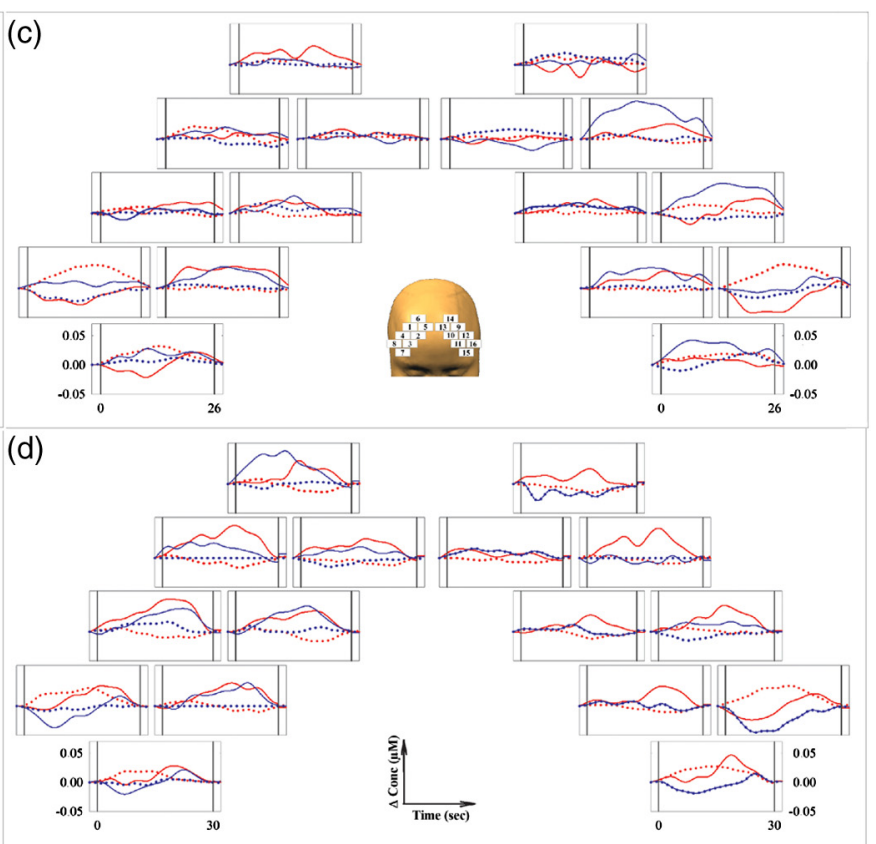

of the BOLD response depends on several factors, and it was suggested that the solution to this problem is a multimodal approach, combining measurements from other techniques including fNIRS. ${ }^{50}$ Unfortunately, in the present study, a combined fNIRS/fMRI measurement was not performed.

The advantages of separated fNIRS measurements have been discussed in detail ${ }^{23,24}$ and can be briefly summarized in three points as follows: (1) the use of low-cost, silent, and transportable/portable instrumentation, making long-term monitoring and repeated measurements of cortical activities possible in various situations, including the natural ones; (2) the measurement of the changes in $\mathrm{O}_{2} \mathrm{Hb}$ and $\mathrm{HHb}$, delivering additional information with respect to the fMRI signal, which in turn is based on the BOLD signal (that is relative only to $\mathrm{HHb}$ changes); and (3) the possibility to move freely during the fNIRS measurements since the subject's body is not fixed as during fMRI/PET measurements.

In the present study, the protocol lasted about $30 \mathrm{~min}$ and the mean subjective rating of the perceived physical and/or psychological discomfort caused by the probe holder mounted on the head was moderate. The fNIRS data could have been corrupted by subtle and merely identifiable motion artifacts and superficial systemic oscillation (that constitute the physiological noise). The fNIRS sensitivity to hemodynamic and oxygenation changes in the superficial compartment is a particular confounding factor of this technique. ${ }^{51}$ To compensate these factors, the "wavelet motion correction" method was applied. Furthermore, to reduce the superficial systemic oscillation, the SS signals were regressed from standard channel signals to avoid physiological confounds to be interpreted as a reliable functional activation of the brain. The method developed by Barker et al. ${ }^{36}$ was chosen to solve the GLM since it considers the serially correlated physiological noise present in the fNIRS data. 
In addition to the differences between these neuroimaging techniques and the strengths of fNIRS, the following differences in tasks and protocols between the present fNIRS study and the previous fMRI study by Toepper et al. ${ }^{13}$ should be taken into consideration: (1) the stimuli presentation times were different: in the fMRI study, times were shorter $(500 \mathrm{~ms})$ than that ones used in this fNIRS study ( $1 \mathrm{~s}$, according to the standard clinical procedure adopted in Italy). ${ }^{37}$ (2) The number of the CBTs and $\mathrm{CBTb}$ sequences was different: 10 and 5 sequences per length were presented in the fMRI and in the fNIRS study, respectively. (3) Subject's spatial span was not assessed in the fMRI study, whereas in the present study, it was assessed both with the computerized CBT and the original CBT.

Among the above-mentioned differences, it is important to highlight that the shorter timing used in Toepper et al. ${ }^{13}$ might require a higher-level of cognitive effort during task execution. All these differences could in part justify the discrepant results between the two studies. Both studies utilized a computerized CBT whose strengths and limitations should be taken into consideration. In recent years, digital technologies have been applied in cognitive performance testing, often replacing traditional versions. However, the extent to which findings from traditional and computerized tasks are equivalent still remains unclear. ${ }^{52}$ In fact, digital testing differs from the noncomputerized one in several important ways. Computerized cognitive tasks are administered as a two-dimensional (2-D) representation of the tasks on a PC screen rather than a three-dimensional (3-D) representation provided by the traditional version. Moreover, traditional and digital cognitive tasks differ in the way in which the participants give a response. When using the 3-D tool, the participant receives a haptic experience, whereas during digital cognitive testing, indirect input devices such as keypad are used. During the execution of traditional version of the CBT, subjects can feel and handle the wood board (the task requires the recall and the reproduction of the spatial cube sequences through hand tapping-movements; Fig. 1). The haptic experience is missing when participants use a keyboard or touch screen in the computerized version. In fact, digital paradigm may require several stepwise choice decisions and reproduction through finger key-pressing movements when using the keypad and a 2-D touch experience when using touch screen apparatus. ${ }^{52}$ When using digital versions of tasks with clinical and/ or research purpose, it is necessary to take into account the different strategies that could be employed when the task is presented in its traditional or computerized form. ${ }^{53}$ One recent study has found a divergent performance pattern (specifically in the backward reproduction) on the two versions of the CBT, resulting in a warning regarding the consequences on the cognitive concepts that it is assumed to assess. ${ }^{54}$ For these reasons, even if some studies have identified no differences in the CBT performance between traditional and computerized forms, ${ }^{52,55}$ a familiarization session was carried out in the present study, both to make subjects confident with the setting and to compare performance resulting from the two task versions. Finally, it is important to mention that in the present study, a computerized version of the CBT was used instead of the original wooden board to standardize the administration timing. It is well known that examiner's timing of administration could affect the subject's CBT performance. ${ }^{19}$

For an adequate understanding of the current findings, some limitations should be pointed out: (1) a connectivity investigation over the PFC was not possible, given the relatively small cortical areas that were monitored with the 16-measurement point fNIRS system. For the same reason, the parietal and occipital cortical areas, although were supposed to play a pivotal role in performing the CBTs and CBTb, were not investigated. Furthermore, systems with a higher number of measurement points (e.g., high-density diffuse optical tomography systems) would allow a higher spatial resolution and a suitable investigation of the different frontal-posterior cortical connections implied in spatial WM processes. (2) The fNIRS evaluates only the cortical area and, for this reason, it was not possible to evaluate the hippocampus and other subcortical areas activation during the CBTs and CBTb execution. (3) Although a haptic experience may benefit CBT execution, the attempt to standardize the administration of the CBT task did not make it possible to include a 3-D CBT board in the experimental setting. (4) No individual structural MRI data were collected to get a precise localization of the 16 cortical fNIRS measurement points. Therefore, the lack of this precise spatial information could make the interpretation of fNIRS results less meaningful.

\section{Conclusion}

Over the last 27 years, several fNIRS studies on WM have provided significant results. Unfortunately, the fNIRS results of the present study have not allowed us to enhance understanding regarding the specific role of the $\mathrm{PFC}$ subregions during the CBT execution. The observed lack of a specific VLPFC and DLPFC involvement is surprising, since the developed computerized version of the CBT was very close to the standard procedure adopted in clinical practice and similar to the CBT version utilized in the recent fMRI study. ${ }^{13}$ Nevertheless, the lack of this specific activation of the VLPFC and DLPFC should be interpreted very carefully and it should serve only as a starting point for future studies, which should be performed for a better understanding of the short-term circuits and WM processes implied during the CBT execution (using both the original and the computerized version of the task). In addition, this investigation could be performed through a multimodal approach or at least through high-density diffuse optical tomography systems to obtain 3-D functional images of the human brain. ${ }^{56-59}$ Neuroimaging results about CBT execution could give important information regarding the effects of the rehabilitation treatment in patients with frontal cortex injuries and visuospatial WM memory impairments.

\section{Disclosures}

None of the authors has any conflicts of interest to disclose.

\section{Acknowledgments}

The study has been performed in the framework of the "Interdepartmental Research Centre for Molecular Diagnostics and Advanced Therapies." This work was supported in part by the "Abruzzo Earthquake Relief Fund" (Toronto, Ontario; purchase of the Artinis system).

\section{References}

1. A. D. Baddeley, G. Hitch, and G. A. Bower, "Working memory," in Recent Advances in Learning and Motivation, Academic Press, New York (1974).

2. A. D. Baddeley, "Working memory and language: an overview," J. Commun. Disord. 36(3), 189-208 (2003). 
3. F. Collette et al., "Exploration of the neural substrates of executive functioning by functional neuroimaging," Neuroscience 139(1), 209-221 (2006).

4. A. A. Hartley and N. K. Speer, "Locating and fractionating working memory using functional neuroimaging: storage, maintenance, and executive functions," Microsc. Res. Technol. 51(1), 45-53 (2000).

5. A. H. Lara and J. D. Wallis, "The role of prefrontal cortex in working memory: a mini review," Front. Syst. Neurosci. 9, 173 (2015).

6. M. Corbetta and G. L. Shulman, "Spatial neglect and attention networks," Аnnu. Rev. Neurosci. 34, 569-599 (2011).

7. P. Malhotra, E. J. Coulthard, and M. Husain, "Role of right posterior parietal cortex in maintaining attention to spatial locations over time," Brain 132(3), 645-660 (2009).

8. T. D. Wager and E. E. Smith, "Neuroimaging studies of working memory: a meta-analysis," Cognit. Affective Behav. Neurosci. 3(4), 255-274 (2003).

9. D. Bor et al., "Frontal lobe involvement in spatial span: converging studies of normal and impaired function," Neuropsychologia 44(2), 229237 (2006).

10. Q. Chen, P. Wei, and X. Zhou, "Distinct neural correlates for resolving stroop conflict at inhibited and noninhibited locations in inhibition of return," J. Cognit. Neurosci. 18(11), 1937-1946 (2006).

11. M. Petrides, "Functional organization of the human frontal cortex for mnemonic processing. Evidence from neuroimaging studies," Ann. N. Y. Acad. Sci. 769, 85-96 (1995).

12. M. Sandrini, P. M. Rossini, and C. Miniussi, "Lateralized contribution of prefrontal cortex in controlling task-irrelevant information during verbal and spatial working memory tasks: rTMS evidence," Neuropsychologia 46(7), 2056-2063 (2008).

13. M. Toepper et al., "Functional correlates of distractor suppression during spatial working memory encoding," Neuroscience 165(4), 1244 1253 (2010).

14. G. Deco, E. T. Rolls, and B. Horwitz, "'What' and 'where' in visual working memory: a computational neurodynamical perspective for integrating fMRI and single-neuron data," J. Cognit. Neurosci. 16(4), 683701 (2004).

15. J. B. Sala, P. Rämä, and S. M. Courtney, "Functional topography of a distributed neural system for spatial and nonspatial information maintenance in working memory," Neuropsychologia 41, 341-356 (2003).

16. L. G. Ungerleider, S. M. Courtney, and J. V. Haxby, "A neural system for human visual working memory," Proc. Natl. Acad. Sci. U. S. A. 95(3), 883-890 (1998).

17. P. M. Corsi, "Human memory and the medial temporal region of the brain," Dissertation Abstract International, Vol. 34, p. 819B (1972).

18. B. Milner, "Clues to the cerebral organization of memory," in Cerebral Correlates of Conscious Experience, INSERM: Symp. No. 6, P. A. Buser and A. Rougeul Buser, Eds., pp. 139-153, Elsevier, North Holland, Amsterdam (1978).

19. D. B. Berch, R. Krikorian, and E. M. Huha, "The Corsi block-tapping task: methodological and theoretical considerations," Brain Cognit. 38(3), 317-338 (1998).

20. T. Beblo et al., "A new approach in clinical neuropsychology to the assessment of spatial working memory: the block suppression test," J. Clin. Exp. Neuropsychol. 26(1), 105-114 (2004).

21. S. Cutini, S. B. Moro, and S. Bisconti, "Functional near infrared optical imaging in cognitive neuroscience: an introductory review," J. Near Infrared Spectrosc. 20(1), 75-92 (2012).

22. A. C. Ehlis et al., "Application of functional near-infrared spectroscopy in psychiatry," Neuroimage $\mathbf{8 5}(1)$, 478-488 (2014).

23. M. Ferrari and V. Quaresima, "A brief review on the history of human functional near-infrared spectroscopy (fNIRS) development and fields of application," Neuroimage 63(2), 921-935 (2012).

24. F. Scholkmann et al., "A review on continuous wave functional nearinfrared spectroscopy and imaging instrumentation and methodology," Neuroimage 85(1), 6-27 (2014).

25. R. Oldfield, "The assessment and analysis of handedness: the Edinburgh inventory," Neuropsychologia 9(1), 97-113 (1971).

26. D. T. Delpy et al., "Estimation of optical pathlength through tissue from direct time of flight measurement," Phys. Med. Biol. 33(12), 1433-1442 (1988).
27. A. Duncan et al., "Measurement of cranial optical path length as a function of age using phase resolved near infrared spectroscopy," Pediatr. Res. 39(5), 889-894 (1996).

28. S. Cutini, P. Scatturin, and M. Zorzi, "A new method based on ICBM152 head surface for probe placement in multichannel fNIRS," Neuroimage 54(2), 919-927 (2011).

29. J. Mazziotta et al., "A probabilistic atlas and reference system for the human brain: international consortium for brain mapping (ICBM)," Philos. Trans. R. Soc., B 356(1412), 1293-1322 (2001).

30. S. Basso Moro et al., "A semi-immersive virtual reality incremental swing balance task activates prefrontal cortex: a functional near-infrared spectroscopy study," Neuroimage 85(1), 451-460 (2014).

31. T. J. Huppert et al., "HomER: a review of time-series analysis methods for near-infrared spectroscopy of the brain," Appl. Opt. 48(10), D280298 (2009).

32. C. M. Aasted et al., "Anatomical guidance for functional near-infrared spectroscopy: atlas viewer tutorial," Neurophotonics 2(2), 020801 (2015).

33. T. Takahashi et al., "Influence of skin blood flow on near-infrared spectroscopy signals measured on the forehead during a verbal fluency task," Neuroimage 57(3), 991-1002 (2011).

34. B. Molavi and G. A. Dumont, "Wavelet-based motion artifact removal for functional near-infrared spectroscopy," Physiol. Meas. 33(2), 259270 (2012).

35. L. Gagnon et al., "Improved recovery of the hemodynamic response in diffuse optical imaging using short optode separations and state-space modelling," Neuroimage 56(3), 1362-1371 (2011).

36. W. Barker, A. Aarabi, and T. Huppert, "Autoregressive model based algorithm for correcting motion and serially correlated errors in fNIRS," Biomed. Opt. Express 4(8), 1366-1379 (2013).

37. M. Monaco et al., "Forward and backward span for verbal and visuospatial data: standardization and normative data from an Italian adult population," Neurol. Sci. 34(5), 749-754 (2013).

38. M. P. Jensen, C. Chen, and A. M. Brugger, "Interpretation of visual analog scale ratings and change scores: a reanalysis of two clinical trials of postoperative pain," J. Pain 4(7), 407-414 (2003).

39. C. D. Spielberger et al., Manual for the State-Trait Anxiety Inventory, Consulting Psychologists Press, Palo Alto (1983).

40. E. J. Wagenmakers et al., "Bayesian inference for psychology. Part II: example applications with JASP," Psychon. Bull. Rev. (2017).

41. S. Arai et al., "Altered frontal pole development affects self-generated spatial working memory in ADHD," Brain Dev. 38(5), 471-480 (2016).

42. J. Lee et al., "Origins of spatial working memory deficits in schizophrenia: an event-related fMRI and near-infrared spectroscopy study," PLoS One 3(3), e1760 (2008).

43. R. McKendrick et al., "Enhancing dual-task performance with verbal and spatial working memory training: continuous monitoring of cerebral hemodynamics with NIRS," Neuroimage 85(3), 1014-1026 (2014).

44. S. B. Perlman, T. J. Huppert, and B. Luna, "Functional near-infrared spectroscopy evidence for development of prefrontal engagement in working memory in early through middle childhood," Cereb. Cortex 26(6), 2790-2799 (2016).

45. H. Ayaz et al., "Continuous monitoring of brain dynamics with functional near infrared spectroscopy as a tool for neuroergonomic research: empirical examples and a technological development," Front. Hum. Neurosci. 7, 871 (2013).

46. A. Vermeij et al., "An exploratory study of the effects of spatial working-memory load on prefrontal activation in low- and high-performing elderly," Front. Aging Neurosci. 6, 303 (2014).

47. D. G. Giacalone et al., "Cerebral time domain-NIRS: reproducibility analysis, optical properties, hemoglobin species and tissue oxygen saturation in a cohort of adult subjects," Biomed. Opt. Express 8(11), 4987-5000 (2017).

48. T. J. Huppert et al., "Comparison of group-level, source localized activity for simultaneous functional near-infrared spectroscopy-magnetoencephalography and simultaneous fNIRS-fMRI during parametric median nerve stimulation," Neurophotonics 4(1), 015001 (2017).

49. J. Steinbrink et al., "Illuminating the BOLD signal: combined fMRIfNIRS studies," Magn. Reson. Imaging 24(4), 495-505 (2006).

50. R. B. Buxton, "The physics of functional magnetic resonance imaging (fMRI)," Rep. Prog. Phys. 76(9), 096601 (2013). 
51. I. Tachtsidis and F. Scholkmann, "False positives and false negatives in functional near-infrared spectroscopy: issues, challenges, and the way forward," Neurophotonics 3(3), 031405 (2016).

52. S. J. Robinson and G. Brewer, "Performance on the traditional and the touch screen, tablet versions of the Corsi block and the tower of Hanoi tasks," Comput. Hum. Behav. 60, 29-34 (2016).

53. J. M. Noyes and K. J. Garland, "Solving the tower of Hanoi: does mode of presentation matter?" Comput. Hum. Behav. 19(5), 579-592 (2003).

54. M. H. Claessen, I. J. van der Ham, and M. J. van Zandvoort, "Computerization of the standard Corsi block-tapping task affects its underlying cognitive concepts: a pilot study," Appl. Neuropsychol. Adult 22(3), 180-188 (2015).

55. R. E. Nelson, A. L. Dickson, and J. H. Baños, "An automated administration of Corsi's block-tapping test," Perceptual Mot. Skills 91(2), 578-580 (2000).

56. M. A. Kamran, M. M. Mannan, and M. Y. Jeong, "Cortical signal analysis and advances in functional near-infrared spectroscopy signal: a review," Front. Hum. Neurosci. 10, 261 (2016).

57. D. Chitnis et al., "Functional imaging of the human brain using a modular, fibre-less, high-density diffuse optical tomography system," Biomed. Opt. Express 7(10), 4275 (2016).

58. A. T. Eggebrecht et al., "Mapping distributed brain function and networks with diffuse optical tomography," Nat. Photonics 8(6), 448454 (2014).

59. H. Zhao and R. J. Cooper, "Review of recent progress toward a fiberless, whole-scalp diffuse optical tomography system," Neurophotonics $\mathbf{5}(1)$, 011012 (2017).

Stefania Lancia is a PhD student at the Department of Life, Health and Environmental Sciences, L'Aquila University, Italy. Her research interest is focused on the investigation of the cerebral cortex hemodynamic changes in response to cognitive tasks revealed by functional near-infrared spectroscopy (fNIRS).

Vincenza Cofini is an assistant professor of medical statistics at the Department of Life, Health and Environmental Sciences, L'Aquila University, Italy. Her main research activity is focused on clinical epidemiology and public health with expertise on design and analysis of randomized clinical trials.

Marika Carrieri is a PhD student at the Department of Life, Health and Environmental Sciences, L'Aquila University, Italy. Her research interest is focused on the investigation of the cerebral cortex hemodynamic changes in response to cognitive tasks revealed by functional near-infrared spectroscopy (fNIRS).

Marco Ferrari is a biochemistry professor at the Department of Life, Health and Environmental Sciences, L'Aquila University, Italy. He has pioneered near-infrared spectroscopy brain applications. His research activity is focused on the study of the cerebral cortex metabolic/hemodynamic changes on cognitive/motor tasks of different complexity by using functional near-infrared spectroscopy (fNIRS).

Valentina Quaresima is a biochemistry professor at the Department of Life, Health and Environmental Sciences, L'Aquila University, Italy. Her research interest currently focuses on the investigation of the cerebral cortex metabolic/hemodynamic changes in response to cognitive and motor tasks of different complexity revealed by functional near-infrared spectroscopy (fNIRS). 